\title{
New Hermite-Hadamard type inequalities for $n$-polynomial harmonically convex functions
}

\author{
Muhammad Uzair Awan', Nousheen Akhtar', Sabah Iftikhar², Muhammad Aslam Noor² and \\ Yu-Ming $\mathrm{Chu}^{3,4^{*}}$ (i)
}

"Correspondence:

chuyuming2005@126.com

${ }^{3}$ Department of Mathematics,

Huzhou University, Huzhou, China

${ }^{4}$ Hunan Provincial Key Laboratory of

Mathematical Modeling and

Analysis in Engineering, Changsha

University of Science \& Technology,

Changsha, China

Full list of author information is

available at the end of the article

\section{Springer}

\begin{abstract}
In the article, we introduce a class of $n$-polynomial harmonically convex functions, establish their several new Hermite-Hadamard type inequalities which are the generalizations and variants of the previously known results for harmonically convex functions.
\end{abstract}

MSC: 26A51;26D10;26D15

Keywords: $n$-polynomial; Harmonic convex function; Hermite-Hadamard inequality

\section{Introduction and preliminaries}

Let $I \subseteq \mathbb{R}$ be an interval. Then a real-valued function $f: I \rightarrow \mathbb{R}$ is said to be convex (concave) if the inequality

$$
f(\lambda x+(1-\lambda) y) \leq(\geq) \lambda f(x)+(1-\lambda) f(y)
$$

holds for all $x, y \in I$ and $\lambda \in[0,1]$.

It is well known that the convexity (concavity) has wide applications in many branches of pure and applied mathematics [1-30], many inequalities can be derived via the convexity or concavity theory [31-56]. Recently, the generalizations, extensions, and variants for the convexity have attracted the attention of many researchers [57-65].

The classical Hermite-Hadamard inequality [66] is the most famous one in convex functions theory, which states that the double inequality

$$
f\left(\frac{x+y}{2}\right) \leq(\geq) \frac{1}{y-x} \int_{x}^{y} f(t) d t \leq(\geq) \frac{f(x)+f(y)}{2}
$$

holds for all $x, y \in I$ with $x \neq y$ if $f: I \rightarrow \mathbb{R}$ is a convex (concave) function.

In the past few decades, many generalizations, improvements, refinements, and variants for the Hermite-Hadamard inequality have been made by several researchers, we recommend the literature [67-72] to interested readers.

(c) The Author(s) 2020. This article is licensed under a Creative Commons Attribution 4.0 International License, which permits use, sharing, adaptation, distribution and reproduction in any medium or format, as long as you give appropriate credit to the original author(s) and the source, provide a link to the Creative Commons licence, and indicate if changes were made. The images or other third party material in this article are included in the article's Creative Commons licence, unless indicated otherwise in a credit line to the material. If material is not included in the article's Creative Commons licence and your intended use is not permitted by statutory regulation or exceeds the permitted use, you will need to obtain permission directly from the copyright holder. To view a copy of this licence, visit http://creativecommons.org/licenses/by/4.0/. 
Now, we recall the definition of harmonically convex function [73] as follows.

Definition 1.1 (see [73]) Let $H \subseteq(0, \infty)$ be an interval. Then a real-valued function $f$ : $H \rightarrow \mathbb{R}$ is said to be harmonically convex if

$$
f\left(\frac{x y}{t x+(1-t) y}\right) \leq t f(y)+(1-t) f(x)
$$

for all $x, y \in H$ and $t \in[0,1]$.

İşcan [73] provided the Hermite-Hadamard type inequality for the harmonically convex function.

Theorem 1.2 (see [73]) Let $H \subseteq(0, \infty)$ be an interval and $f: H \rightarrow \mathbb{R}$ be a harmonically convex function. Then the Hermite-Hadamard type inequality

$$
f\left(\frac{2 a b}{a+b}\right) \leq \frac{a b}{b-a} \int_{a}^{b} \frac{f(x)}{x^{2}} d x \leq \frac{f(a)+f(b)}{2}
$$

holds for all $a, b \in H$ with $a<b$ iff $\in L[a, b]$.

Very recently, Toplu et al. [74] introduced and investigated a new class of $n$-polynomial convex functions and established several new Hermite-Hadamard type inequalities for this class of functions.

The main purpose of the article is to introduce the notion of $n$-polynomial harmonically convex functions, derive the variants of the classical Hermite-Hadamard inequality by use of the class of $n$-polynomial harmonically convex functions. We also discuss several new special cases for the obtained results which show that our obtained results are the generalizations and extensions of some previously known results.

\section{Results and discussions}

In this section, we first introduce the definition of $n$-polynomial harmonically convex function.

Definition 2.1 Let $n \in \mathbb{N}$ and $H \subseteq(0, \infty)$ be an interval. Then a nonnegative real-valued function $f: H \rightarrow[0, \infty)$ is said to be an $n$-polynomial harmonically convex function if

$$
f\left(\frac{x y}{t x+(1-t) y}\right) \leq \frac{1}{n} \sum_{s=1}^{n}\left(1-(1-t)^{s}\right) f(y)+\frac{1}{n} \sum_{s=1}^{n}\left(1-t^{s}\right) f(x)
$$

for all $x, y \in H$ and $t \in[0,1]$.

From Definitions 1.1 and 2.1, we clearly see that the class of $n$-polynomial harmonically convex functions reduces to the class of harmonically convex functions if $n=1$ and the 2-polynomial harmonically convex function $f$ satisfies the inequality

$$
f\left(\frac{x y}{t x+(1-t) y}\right) \leq \frac{3 t-t^{2}}{2} f(y)+\frac{2-t-t^{2}}{2} f(x) .
$$


Theorem 2.2 Let $b>a>0, f_{\alpha}:[a, b] \rightarrow[0, \infty)$ be a family of n-polynomial harmonically convex functions and $f(u)=\sup _{\alpha} f_{\alpha}(u)$. Then $f$ is an n-polynomial harmonically convex function if $J=\{x \in[a, b]: f(x)<\infty\}$ is an interval.

Proof Let $t \in[0,1]$ and $x, y \in J$. Then we clearly see that

$$
\begin{aligned}
f\left(\frac{x y}{t x+(1-t) y}\right) & =\sup _{\alpha} f_{\alpha}\left(\frac{x y}{t x+(1-t) y}\right) \\
& \leq \frac{1}{n} \sum_{s=1}^{n}\left(1-(1-t)^{s}\right) \sup _{\alpha} f_{\alpha}(y)+\frac{1}{n} \sum_{s=1}^{n}\left(1-t^{s}\right) \sup _{\alpha} f_{\alpha}(x) \\
& =\frac{1}{n} \sum_{s=1}^{n}\left(1-(1-t)^{s}\right) f(y)+\frac{1}{n} \sum_{s=1}^{n}\left(1-t^{s}\right) f(x)<\infty
\end{aligned}
$$

which completes the proof.

Theorem 2.3 Let $f:[a, b] \subseteq(0, \infty) \rightarrow[0, \infty)$ be an n-polynomial harmonically convex function. Then one has

$$
\frac{1}{2}\left(\frac{n}{n+2^{-n}-1}\right) f\left(\frac{2 a b}{a+b}\right) \leq \frac{a b}{b-a} \int_{a}^{b} \frac{f(x)}{x^{2}} d x \leq\left(\frac{f(a)+f(b)}{n}\right) \sum_{s=1}^{n} \frac{s}{s+1}
$$

iff $\in L[a, b]$.

Proof It follows from the $n$-polynomial harmonic convexity of $f$ that

$$
f\left(\frac{x y}{t x+(1-t) y}\right) \leq \frac{1}{n} \sum_{s=1}^{n}\left[1-(1-t)^{s}\right] f(y)+\frac{1}{n} \sum_{s=1}^{n}\left(1-t^{s}\right) f(x),
$$

which leads to

$$
f\left(\frac{2 x y}{x+y}\right) \leq \frac{1}{n} \sum_{s=1}^{n}\left[1-\left(\frac{1}{2}\right)^{s}\right] f(y)+\frac{1}{n} \sum_{s=1}^{n}\left[1-\left(\frac{1}{2}\right)^{s}\right] f(x) .
$$

Using the change of variables, we have

$$
f\left(\frac{2 a b}{a+b}\right) \leq \frac{1}{n} \sum_{s=1}^{n}\left[1-\left(\frac{1}{2}\right)^{s}\right]\left[f\left(\frac{a b}{t a+(1-t) b}\right)+f\left(\frac{a b}{t b+(1-t) a}\right)\right]
$$

Integrating both sides of the above inequality with respect to $t$ on $[0,1]$, we get

$$
\frac{1}{2}\left(\frac{n}{n+2^{-n}-1}\right) f\left(\frac{2 a b}{a+b}\right) \leq \frac{a b}{b-a} \int_{a}^{b} \frac{f(x)}{x^{2}} d x
$$

Note that

$$
\frac{a b}{b-a} \int_{a}^{b} \frac{f(x)}{x^{2}} d x=\int_{o}^{1} f\left(\frac{a b}{t a+(1-t) b}\right) d t
$$




$$
\begin{aligned}
& \leq \int_{0}^{1}\left[\frac{1}{n} \sum_{s=1}^{n}\left[1-(1-t)^{s}\right] f(b)+\frac{1}{n} \sum_{s=1}^{n}\left[1-t^{s}\right] f(a)\right] d t \\
& =\frac{f(b)}{n} \sum_{s=1}^{n} \int_{0}^{1}\left[1-(1-t)^{s}\right] d t+\frac{f(a)}{n} \sum_{s=1}^{n} \int_{0}^{1}\left[1-t^{s}\right] d t \\
& =\left[\frac{f(a)+f(b)}{n}\right] \sum_{s=1}^{n} \frac{s}{s+1} .
\end{aligned}
$$

Therefore, Theorem 2.3 follows from (2.1) and (2.2).

Remark 2.4 Let $n=1$. Then Theorem 2.3 leads to the Hermite-Hadamard inequality for harmonically convex functions of [73].

Lemma 2.5 Let $f:[a, b] \subseteq(0, \infty) \rightarrow \mathbb{R}$ be a differentiable function. Then the identity

$$
\begin{gathered}
\frac{\lambda f(a)+\mu f(b)}{2}+\frac{2-\lambda-\mu}{2} f\left(\frac{2 a b}{a+b}\right)-\frac{a b}{b-a} \int_{a}^{b} \frac{f(x)}{x^{2}} d x \\
=\frac{a b(b-a)}{4} \int_{0}^{1}\left[\frac{4(1-\lambda-t)}{((1-t) a+(1+t) b)^{2}} f^{\prime}\left(\frac{2 a b}{(1-t) a+(1+t) b}\right)\right. \\
\left.+\frac{4(\mu-t)}{((2-t) a+t b)^{2}} f^{\prime}\left(\frac{2 a b}{(2-t) a+t b}\right)\right] d t
\end{gathered}
$$

holds for $\lambda, \mu \in[0,1]$ if $f^{\prime} \in L[a, b]$.

Proof Integrating by parts and changing variable of the definite integral give

$$
\begin{aligned}
I_{1}= & \int_{0}^{1}\left[\frac{4(1-\lambda-t)}{((1-t) a+(1+t) b)^{2}} f^{\prime}\left(\frac{2 a b}{(1-t) a+(1+t) b}\right)\right] d t \\
= & -\frac{2}{a b(b-a)}\left[\left.(1-\lambda-t) f\left(\frac{2 a b}{(1-t) a+(1+t) b}\right)\right|_{0} ^{1}\right. \\
& \left.+\int_{0}^{1} f\left(\frac{2 a b}{(1-t) a+(1+t) b}\right) d t\right] \\
= & \frac{2}{a b(b-a)}\left[\lambda f(a)+(1-\lambda) f\left(\frac{2 a b}{a+b}\right)\right]-\frac{4}{(b-a)^{2}} \int_{a}^{\frac{2 a b}{b-a}} \frac{f(x)}{x^{2}} d x
\end{aligned}
$$

Similarly, we have

$$
\begin{aligned}
I_{2} & =\int_{0}^{1} \frac{4(\mu-t)}{((2-t) a+t b)^{2}} f^{\prime}\left(\frac{2 a b}{(2-t) a+t b)}\right) d t \\
& =-\frac{2}{a b(b-a)}\left[\left.(\mu-t) f\left(\frac{2 a b}{(2-t) a+t b}\right)\right|_{0} ^{1}+\int_{0}^{1} f\left(\frac{2 a b}{(2-t) a+t b}\right) d t\right] \\
& =\frac{2}{a b(b-a)}\left[(1-\mu) f\left(\frac{2 a b}{a+b}\right)+\mu f(b)\right]-\frac{4}{(b-a)^{2}} \int_{\frac{2 a b}{a+b}}^{b} \frac{f(x)}{x^{2}} d x .
\end{aligned}
$$

Multiplying $I_{1}$ and $I_{2}$ by $\frac{a b(b-a)}{4}$ and combining both equalities, we get the desired result. 
For the sake of simplicity, in what follows we denote

$$
A_{a}=((1-t) a+(1+t) b)
$$

and

$$
A_{b}=((2-t) a+t b) .
$$

Before we give our next result, let us recall the definitions of the gamma function $\Gamma(\cdot)$, beta function $B(\cdot, \cdot)$, and hypergeometric function ${ }_{2} F_{1}(\cdot, \cdot ; \cdot ; \cdot)$ as follows:

$$
\begin{aligned}
& \Gamma(x)=\int_{0}^{\infty} e^{-t} t^{x-1} d t, \\
& B(x, y)=\int_{0}^{1} t^{x-1}(1-t)^{y-1} d t, \\
& B(x, y)=\frac{\Gamma(x) \Gamma(y)}{\Gamma(x+y)}, \\
& { }_{2} F_{1}(x, y ; c ; z)=\frac{1}{\mathrm{~B}(y, c-y)} \int_{0}^{1} t^{y-1}(1-t)^{c-y-1}(1-z t)^{-x} d t .
\end{aligned}
$$

Theorem 2.6 Let $p, q>1$ with $1 / p+1 / q=1, \lambda, \mu \in[0,1]$, and $f:[a, b] \subseteq(0, \infty) \rightarrow \mathbb{R}$ be a differentiable function such that $f^{\prime} \in L[a, b]$ and $\left|f^{\prime}\right|^{q}$ is an n-polynomial harmonically convex function. Then we have

$$
\begin{aligned}
& \left|\frac{\lambda f(a)+\mu f(b)}{2}+\frac{2-\lambda-\mu}{2} f\left(\frac{2 a b}{a+b}\right)-\frac{a b}{b-a} \int_{a}^{b} \frac{f(x)}{x^{2}} d x\right| \\
& \leq \frac{a b(b-a)}{4}\left[\left\{\psi_{1}^{\frac{1}{p}}\left(C_{1}\left|f^{\prime}(a)\right|^{q}+C_{2}\left|f^{\prime}(b)\right|^{q}\right)^{\frac{1}{q}}\right\}\right. \\
& \left.\quad+\left\{\psi_{2}^{\frac{1}{p}}\left(C_{3}\left|f^{\prime}(a)\right|^{q}+C_{4}\left|f^{\prime}(b)\right|^{q}\right)^{\frac{1}{q}}\right\}\right],
\end{aligned}
$$

where

$$
\begin{aligned}
\psi_{1}= & 4 \int_{0}^{1}|1-\lambda-t|^{p} d t=4\left(\frac{(1-\lambda)^{p+1}+(\lambda)^{p+1}}{p+1}\right), \\
\psi_{2}= & 4 \int_{0}^{1}|\mu-t|^{p} d t=4\left(\frac{(1-\mu)^{p+1}+(\mu)^{p+1}}{p+1}\right), \\
C_{1}= & \frac{1}{2 n} \sum_{s=1}^{n}\left(2(a+b)^{-2 q}{ }_{2} F_{1}\left(2 q, 1 ; 2 ; \frac{a-b}{a+b}\right)\right. \\
& \left.-\frac{(a+b)^{-2 q}}{s+1}{ }_{2} F_{1}\left(2 q, 1 ; s+2 ; \frac{a-b}{a+b}\right)\right), \\
C_{2}= & \frac{1}{2 n} \sum_{s=1}^{n}\left((a+b)^{-2 q}{ }_{2} F_{1}\left(2 q, 1 ; 2 ; \frac{a-b}{a+b}\right)\right. \\
& \left.-\frac{(a+b)^{-2 q}}{s+1}{ }_{2} F_{1}\left(2 q, s+1 ; s+2 ; \frac{a-b}{a+b}\right)\right),
\end{aligned}
$$




$$
\begin{aligned}
C_{3}= & \frac{1}{2 n} \sum_{s=1}^{n}\left((2 a)^{-2 q}{ }_{2} F_{1}\left(2 q, 1 ; 2 ; \frac{a-b}{2 a}\right)\right. \\
& \left.-\frac{(2 a)^{-2 q}}{s+1}{ }_{2} F_{1}\left(2 q, 1 ; s+2 ; \frac{a-b}{2 a}\right)\right), \\
C_{4}= & \frac{1}{2 n} \sum_{s=1}^{n}\left(2(2 a)^{-2 q}{ }_{2} F_{1}\left(2 q, 1 ; 2 ; \frac{a-b}{2 a}\right)\right. \\
& \left.-\frac{(2 a)^{-2 q}}{s+1}{ }_{2} F_{1}\left(2 q, s+1 ; s+2 ; \frac{a-b}{2 a}\right)\right) .
\end{aligned}
$$

Proof It follows from Lemma 2.5 and Hölder's integral inequality together with the $n$ polynomial harmonic convexity of $\left|f^{\prime}\right|^{q}$ that

$$
\begin{aligned}
& \left|\frac{\lambda f(a)+\mu f(b)}{2}+\frac{(2-\lambda-\mu)}{2} f\left(\frac{2 a b}{a+b}\right)-\frac{a b}{b-a} \int_{a}^{b} \frac{f(x)}{x^{2}} d x\right| \\
& \leq \frac{a b(b-a)}{4}\left[\int_{0}^{1}\left|\frac{4(1-\lambda-t)}{((1-t) a+(1+t) b)^{2}}\right| f^{\prime}\left(\frac{2 a b}{((1-t) a+(1+t) b)}\right) \mid d t\right. \\
& \left.\quad+\int_{0}^{1}\left|\frac{4(\mu-t)}{((2-t) a+t b)^{2}}\right|\left|f^{\prime}\left(\frac{2 a b}{(2-t) a+t b)}\right)\right| d t\right] \\
& \leq \frac{a b(b-a)}{4}\left\{\left(\int_{0}^{1} 4(1-\lambda-t)^{p} d t\right)^{\frac{1}{p}}\right. \\
& \quad \times\left[\int_{0}^{1} \frac{1}{A_{a}^{2 q}}\left(\frac{1}{2 n} \sum_{s=1}^{n}\left[2-(1-t)^{s}\right]\left|f^{\prime}(a)\right|^{q}+\frac{1}{2 n} \sum_{s=1}^{n}\left[1-t^{s}\right]\left|f^{\prime}(b)\right|^{q}\right) d t\right]^{\frac{1}{q}} \\
& \quad+\left(\int_{0}^{1} 4(\mu-t)^{p} \mathrm{~d} t\right)^{\frac{1}{p}} \\
& \left.\quad \times\left[\int_{0}^{1} \frac{1}{A_{b}^{2 q}}\left(\frac{1}{2 n} \sum_{s=1}^{n}\left[1-(1-t)^{s}\right]\left|f^{\prime}(a)\right|^{q}+\frac{1}{2 n} \sum_{s=1}^{n}\left[2-t^{s}\right]\left|f^{\prime}(b)\right|^{q}\right) \mathrm{d} t\right]^{\frac{1}{q}}\right\} \\
& =\frac{a b(b-a)}{4}\left\{\left(\psi_{1}^{\frac{1}{p}}\left(C_{1}\left|f^{\prime}(a)\right|^{q}+C_{2}\left|f^{\prime}(b)\right|\right)^{\frac{1}{q}}\right)\right. \\
& \left.\quad+\left(\psi_{2}^{\frac{1}{p}}\left(C_{3}\left|f^{\prime}(a)\right|^{q}+C_{4}\left|f^{\prime}(b)\right|^{q}\right)^{\frac{1}{q}}\right)\right\} .
\end{aligned}
$$

This completes the proof.

From Theorem 2.6 we get the following Corollaries 2.7 and 2.8 immediately.

Corollary 2.7 Let $\lambda=\mu$. Then Theorem 2.6 leads to the conclusion that

$$
\begin{aligned}
& \left|\frac{\lambda f(a)+\lambda f(b)}{2}+(1-\lambda) f\left(\frac{2 a b}{a+b}\right)-\frac{a b}{b-a} \int_{a}^{b} \frac{f(x)}{x^{2}} d x\right| \\
& \quad \leq \frac{a b(b-a)}{4} \psi_{1}^{\frac{1}{p}}\left[\left\{C_{1}\left|f^{\prime}(a)\right|^{q}+C_{2}\left|f^{\prime}(b)\right|^{q}\right\}^{\frac{1}{q}}+\left\{C_{3}\left|f^{\prime}(a)\right|^{q}+C_{4}\left|f^{\prime}(b)\right|^{q}\right\}^{\frac{1}{q}}\right],
\end{aligned}
$$

where $\psi_{1}, C_{1}, C_{2}, C_{3}$, and $C_{4}$ are given by (2.3), (2.5), (2.6), (2.7), and (2.8), respectively. 
Corollary 2.8 Let $\lambda=\mu=1 / 2$ and $\lambda=\mu=1 / 3$. Then Theorem 2.6 leads to

$$
\begin{aligned}
& \left|\frac{(f(a)+f(b)}{4}+\frac{1}{2} f\left(\frac{2 a b}{a+b}\right)-\frac{a b}{b-a} \int_{a}^{b} \frac{f(x)}{x^{2}} d x\right| \\
& \leq \frac{a b(b-a)}{4}\left(\frac{8^{\frac{1}{p}}}{2^{1+\frac{1}{p}}(p+1)^{\frac{1}{p}}}\right)\left[\left\{C_{1}\left|f^{\prime}(a)\right|^{q}+C_{2}\left|f^{\prime}(b)\right|^{q}\right\}^{\frac{1}{q}}\right. \\
& \left.\quad+\left\{C_{3}\left|f^{\prime}(a)\right|^{q}+C_{4}\left|f^{\prime}(b)\right|^{q}\right\}^{\frac{1}{q}}\right]
\end{aligned}
$$

and

$$
\begin{aligned}
& \left|\frac{(f(a)+f(b)}{6}+\frac{2}{3} f\left(\frac{2 a b}{a+b}\right)-\frac{a b}{b-a} \int_{a}^{b} \frac{f(x)}{x^{2}} d x\right| \\
& \leq \frac{a b(b-a)}{4}\left(4\left(\frac{\left(\frac{2}{3}\right)^{p+1}+\left(\frac{1}{3}\right)^{p+1}}{(p+1)}\right)\right)^{\frac{1}{p}}\left[\left\{C_{1}\left|f^{\prime}(a)\right|^{q}+C_{2}\left|f^{\prime}(b)\right|^{q}\right\}^{\frac{1}{q}}\right. \\
& \left.\quad+\left\{C_{3}\left|f^{\prime}(a)\right|^{q}+C_{4}\left|f^{\prime}(b)\right|^{q}\right\}^{\frac{1}{q}}\right],
\end{aligned}
$$

where $C_{1}, C_{2}, C_{3}$, and $C_{4}$ are given by (2.5), (2.6), (2.7), and (2.8), respectively.

Theorem 2.9 Let $p, q>1$ with $1 / p+1 / q=1, \lambda, \mu \in[0,1]$, and $f:[a, b] \subseteq(0, \infty) \rightarrow \mathbb{R}$ be a differentiable function such that $f^{\prime} \in L[a, b]$ and $\left|f^{\prime}\right|^{q}$ is an n-polynomial harmonically convex function. Then

$$
\begin{aligned}
& \left|\frac{\lambda f(a)+\mu f(b)}{2}+\frac{2-\lambda-\mu}{2} f\left(\frac{2 a b}{a+b}\right)-\frac{a b}{b-a} \int_{a}^{b} \frac{f(x)}{x^{2}} d x\right| \\
& \leq \frac{a b(b-a)}{4}\left[\frac{4}{(a+b)^{2}}\left({ }_{2} F_{1}\left(2 p, 1 ; 2 ; \frac{a-b}{a+b}\right)\right)^{\frac{1}{p}}\left(C_{5}\left|f^{\prime}(a)\right|^{q}+C_{6}\left|f^{\prime}(b)\right|^{q}\right)^{\frac{1}{q}}\right. \\
& \left.\quad+\frac{1}{a^{2}}\left({ }_{2} F_{1}\left(2 p, 1 ; 2 ; \frac{a-b}{2 a}\right)\right)^{\frac{1}{p}}\left(C_{7}\left|f^{\prime}(a)\right|^{q}+C_{8}\left|f^{\prime}(b)\right|^{q}\right)^{\frac{1}{q}}\right],
\end{aligned}
$$

where

$$
\begin{aligned}
& C_{5}=\frac{1}{2 n} \sum_{s=1}^{n}\left(2 \frac{(1-\lambda)^{q+1}+\lambda q^{q+1}}{q+1}-\int_{0}^{1}|1-\lambda-t|^{q}(1-t)^{s} d t\right), \\
& C_{6}=\frac{1}{2 n} \sum_{s=1}^{n}\left(\frac{(1-\lambda)^{q+1}+\lambda^{q+1}}{q+1}-\int_{0}^{1}|1-\lambda-t|^{q} t^{s} d t\right), \\
& C_{7}=\frac{1}{2 n} \sum_{s=1}^{n}\left(\frac{(1-\mu)^{q+1}+\mu^{q+1}}{q+1}-\int_{0}^{1}|\mu-t|^{q}(1-t)^{s} d t\right), \\
& C_{8}=\frac{1}{2 n} \sum_{s=1}^{n}\left(2 \frac{(1-\mu)^{q+1}+\mu^{q+1}}{q+1}-\int_{0}^{1}|\mu-t|^{q} t^{s} d t\right) .
\end{aligned}
$$


Proof Using Lemma 2.5, Hölder's integral inequality, and the $n$-polynomial harmonic convexity of $\left|f^{\prime}\right|^{q}$, we have

$$
\begin{aligned}
\mid \frac{\lambda f(a)}{2}+\mu f(b) & +\frac{(2-\lambda-\mu)}{2} f\left(\frac{2 a b}{a+b}\right)-\frac{a b}{b-a} \int_{a}^{b} \frac{f(x)}{x^{2}} d x \mid \\
\leq & \frac{a b(b-a)}{4}\left[\int_{0}^{1}\left|\frac{4(1-\lambda-t)}{((1-t) a+(1+t) b)^{2}}\right|\left|f^{\prime}\left(\frac{2 a b}{((1-t) a+(1+t) b)}\right)\right| d t\right. \\
& \left.+\int_{0}^{1}\left|\frac{4(\mu-t)}{((2-t) a+t b)^{2}}\right|\left|f^{\prime}\left(\frac{2 a b}{(2-t) a+t b)}\right)\right| d t\right] \\
\leq & \frac{a b(b-a)}{4}\left\{4\left(\int_{0}^{1} \frac{1}{A_{a}^{2 p}} d t\right)^{\frac{1}{p}}\right. \\
& \times\left[\int_{0}^{1}|1-\lambda-t|^{q}\left(\frac{1}{2 n} \sum_{s=1}^{n}\left[2-(1-t)^{s}\right]\left|f^{\prime}(a)\right|^{q}+\frac{1}{2 n} \sum_{s=1}^{n}\left[1-t^{s}\right]\left|f^{\prime}(b)\right|^{q}\right) d t\right. \\
& +4\left(\int_{0}^{1} \frac{1}{A_{b}^{2 p}} d t\right)^{\frac{1}{p}} \\
& \left.+\frac{1}{a^{2}}\left({ }_{2} F_{1}\left(2 p, 1 ; 2 ; \frac{a-b}{2 a}\right)\right)^{\frac{1}{p}}\left(C_{7}\left|f^{\prime}(a)\right|^{q}+C_{8}\left|f^{\prime}(b)\right|^{q}\right)^{\frac{1}{q}}\right] . \\
\quad & \left.\left.\frac{a b(b-a)}{4}\left[\frac{4}{(a+b)^{2}}\left({ }_{2} F_{1}\left(2 p, 1 ; 2 ; \frac{a-b}{a+b}\right)\right)^{\frac{1}{p}}\left(C_{5}\left|f^{\prime}(a)\right|^{q}+C_{6}\left|f^{\prime}(b)\right|^{q}\right)^{\frac{1}{q}} \sum_{s=1}^{n}\left[1-(1-t)^{s}\right]\left|f^{\prime}(a)\right|^{q}+\frac{1}{2 n} \sum_{s=1}^{n}\left[2-t^{s}\right]\left|f^{\prime}(b)\right|^{q}\right)\right]^{\frac{1}{q}}\right\}
\end{aligned}
$$

This completes the proof.

Remark 2.10 Let $n=1$. Then inequality (2.9) reduces to the following inequality for harmonically convex function:

$$
\begin{aligned}
& \left|\frac{\lambda f(a)+\mu f(b)}{2}+\frac{2-\lambda-\mu}{2} f\left(\frac{2 a b}{a+b}\right)-\frac{a b}{b-a} \int_{a}^{b} \frac{f(x)}{x^{2}} d x\right| \\
& \leq \frac{a b(b-a)}{4}\left[\frac{4}{(a+b)^{2}}\left({ }_{2} F_{1}\left(2 p, 1 ; 2 ; \frac{a-b}{a+b}\right)\right)^{\frac{1}{p}}\left(C_{5}^{*}\left|f^{\prime}(a)\right|^{q}+C_{6}^{*}\left|f^{\prime}(b)\right|^{q}\right)^{\frac{1}{q}}\right. \\
& \left.\quad+\frac{1}{a^{2}}\left({ }_{2} F_{1}\left(2 p, 1 ; 2 ; \frac{a-b}{2 a}\right)\right)^{\frac{1}{p}}\left(C_{7}^{*}\left|f^{\prime}(a)\right|^{q}+C_{8}^{*}\left|f^{\prime}(b)\right|^{q}\right)^{\frac{1}{q}}\right],
\end{aligned}
$$

where

$$
\begin{aligned}
C_{5}^{*} & =\frac{1}{2} \int_{0}^{1}|1-\lambda-t|^{q}(1+t) d t \\
& =\frac{1}{2}\left(\frac{(1-\lambda)^{q+1}+(\lambda)^{q+1}}{q+1}+\frac{(1-\lambda)^{q+2}+(q+2-\lambda)(\lambda)^{q+1}}{(q+1)(q+2)}\right) \\
& =\frac{1}{2(q+1)(q+2)}\left((q+3-\lambda)(1-\lambda)^{q+1}+(2 q+4-\lambda) \lambda^{q+1}\right),
\end{aligned}
$$




$$
\begin{aligned}
C_{6}^{*} & =\frac{1}{2} \int_{0}^{1}|1-\lambda-t|^{q}(1-t) d t \\
& =\frac{1}{2}\left(\frac{(1-\lambda)^{q+1}+(\lambda)^{q+1}}{q+1}-\frac{(1-\lambda)^{q+2}+(q+2-\lambda)(\lambda)^{q+1}}{(q+1)(q+2)}\right) \\
& =\frac{1}{2(q+1)(q+2)}\left((q+1+\lambda)(1-\lambda)^{q+1}+\lambda^{q+2}\right), \\
C_{7}^{*} & =\frac{1}{2} \sum_{s=1}^{n} \int_{0}^{1}|\mu-t|^{q} t d t=\frac{1}{2(q+1)(q+2)}\left((\mu)^{q+2}+(q+1+\mu)(1-\mu)^{q+1}\right), \\
C_{8}^{*} & =\frac{1}{2} \sum_{s=1}^{n} \int_{0}^{1}|\mu-t|^{q}(2-t) d t \\
& =\frac{1}{2}\left(2\left(\frac{(\mu)^{q+1}+(1-\mu)^{q+1}}{q+1}\right)-\frac{(\mu)^{q+2}+(q+1+\mu)(1-\mu)^{q+1}}{(q+1)(q+2)}\right) \\
& =\frac{1}{2(q+1)(q+2)}\left((q+3-\mu)(1-\mu)^{q+1}+(2 q+4-\mu) \mu^{q+1}\right) .
\end{aligned}
$$

Remark 2.11 If $q=1$ and $n=1$, then Theorem 2.9 reduces to

$$
\begin{aligned}
& \left|\frac{\lambda f(a)+\mu f(b)}{2}+\frac{2-\lambda-\mu}{2} f\left(\frac{2 a b}{a+b}\right)-\frac{a b}{b-a} \int_{a}^{b} \frac{f(x)}{x^{2}} d x\right| \\
& \leq \frac{a b(b-a)}{4}\left[\frac{4}{(a+b)^{2}}\left({ }_{2} F_{1}\left(2 p, 1 ; 2 ; \frac{a-b}{a+b}\right)\right)^{\frac{1}{p}}\left(C_{5}^{* *}\left|f^{\prime}(a)\right|+C_{6}^{* *}\left|f^{\prime}(b)\right|\right)\right. \\
& \left.\quad+\frac{1}{a^{2}}\left({ }_{2} F_{1}\left(2 p, 1 ; 2 ; \frac{a-b}{2 a}\right)\right)^{\frac{1}{p}}\left(C_{7}^{* *}\left|f^{\prime}(a)\right|+C_{8}^{* *}\left|f^{\prime}(b)\right|\right)\right],
\end{aligned}
$$

where

$$
\begin{aligned}
& C_{5}^{* *}=\frac{1}{12}\left[(4-\lambda)(1-\lambda)^{2}+(6-\lambda) \lambda^{2}\right], \\
& C_{6}^{* *}=\frac{1}{12}\left[(2+\lambda)(1-\lambda)^{2}+\lambda^{3}\right], \\
& C_{7}^{* *}=\frac{1}{12}\left[(2+\mu)(1-\mu)^{2}+\mu^{3}\right], \\
& C_{8}^{* *}=\frac{1}{12}\left[(4-\mu)(1-\mu)^{2}+(6-\mu) \mu^{2}\right] .
\end{aligned}
$$

Remark 2.12 If $q=1, n=1$, and $\lambda=\mu$, then inequality (2.9) becomes

$$
\begin{aligned}
& \left|\frac{\lambda f(a)+\lambda f(b)}{2}+(1-\lambda) f\left(\frac{2 a b}{a+b}\right)-\frac{a b}{b-a} \int_{a}^{b} \frac{f(x)}{x^{2}} d x\right| \\
& \leq \frac{a b(b-a)}{4}\left[\frac{4}{(a+b)^{2}}\left({ }_{2} F_{1}\left(2 p, 1 ; 2 ; \frac{a-b}{a+b}\right)\right)^{\frac{1}{p}}\left(C_{5}^{* *}\left|f^{\prime}(a)\right|+C_{6}^{* *}\left|f^{\prime}(b)\right|\right)\right. \\
& \left.\quad+\frac{1}{a^{2}}\left({ }_{2} F_{1}\left(2 p, 1 ; 2 ; \frac{a-b}{2 a}\right)\right)^{\frac{1}{p}}\left(C_{6}^{* *}\left|f^{\prime}(a)\right|+C_{5}^{* *}\left|f^{\prime}(b)\right|\right)\right] .
\end{aligned}
$$


Remark 2.13 Let $q=1, n=1$, and $\lambda=\mu=1 / 2$. Then Theorem 2.9 leads to the conclusion that

$$
\begin{aligned}
& \left|\frac{f(a)+f(b)}{4}+\frac{1}{2} f\left(\frac{2 a b}{a+b}\right)-\frac{a b}{b-a} \int_{a}^{b} \frac{f(x)}{x^{2}} d x\right| \\
& \leq \frac{a b(b-a)}{384}\left[\frac{4}{(a+b)^{2}}\left({ }_{2} F_{1}\left(2 p, 1 ; 2 ; \frac{a-b}{a+b}\right)\right)^{\frac{1}{p}}\left(18\left|f^{\prime}(a)\right|+6\left|f^{\prime}(b)\right|\right)\right. \\
& \left.\quad+\frac{1}{a^{2}}\left({ }_{2} F_{1}\left(2 p, 1 ; 2 ; \frac{a-b}{2 a}\right)\right)^{\frac{1}{p}}\left(6\left|f^{\prime}(a)\right|+18\left|f^{\prime}(b)\right|\right)\right] .
\end{aligned}
$$

\section{Conclusion}

In this paper, we have introduced a new class of harmonically convex functions, which are called $n$-polynomial harmonically convex functions, derived several new versions of the Hermite-Hadamard inequality using the class of $n$-polynomial harmonically convex functions and a new integral identity for the differentiable function. We have also discussed some special cases of the obtained results which show that our results are the generalizations and extensions of some previously known results for the harmonically convex functions. Our ideas and approach may lead to a lot of follow-up research.

\section{Acknowledgements}

The authors would like to express their sincere thanks to the editor and the anonymous reviewers for their helpful comments and suggestions.

\section{Funding}

The work was supported by the Natural Science Foundation of China (Grant Nos. 61673169, 11301127, 11701176, $11626101,11601485)$.

Availability of data and materials

Not applicable.

\section{Competing interests}

The authors declare that they have no competing interests.

\section{Authors' contributions}

All authors contributed equally to the writing of this paper. All authors read and approved the final manuscript.

\section{Author details}

${ }^{1}$ Department of Mathematics, Government College University, Faisalabad, Pakistan. ${ }^{2}$ Department of Mathematics, COMSATS University Islamabad, Islamabad, Pakistan. ${ }^{3}$ Department of Mathematics, Huzhou University, Huzhou, China. ${ }^{4}$ Hunan Provincial Key Laboratory of Mathematical Modeling and Analysis in Engineering, Changsha University of Science \& Technology, Changsha, China.

\section{Publisher's Note}

Springer Nature remains neutral with regard to jurisdictional claims in published maps and institutional affiliations.

Received: 18 February 2020 Accepted: 23 April 2020 Published online: 06 May 2020

\section{References}

1. Khan, S., Adil Khan, M., Chu, Y.-M.: New converses of Jensen inequalities via Green functions with applications. Rev. R. Acad. Cienc. Exactas Fís. Nat., Ser. A Mat. 114, Article ID 114 (2020). https://doi.org/10.1007/s13398-020-00843-1

2. Wang, M.-K., Hong, M.-Y., Xu, Y.-F., Shen, Z.-H., Chu, Y.-M.: Inequalities for generalized trigonometric and hyperbolic functions with one parameter. J. Math. Inequal. 14(1), 1-21 (2020)

3. Zhao, T.-H., Shi, L., Chu, Y.-M.: Convexity and concavity of the modified Bessel functions of the first kind with respect to Hölder means. Rev. R. Acad. Cienc. Exactas Fís. Nat., Ser. A Mat. 114(2), Article ID 96 (2020). https://doi.org/10.1007/s13398-020-00825-3

4. Wang, M.-K., He, Z.-Y., Chu, Y.-M.: Sharp power mean inequalities for the generalized elliptic integral of the first kind. Comput. Methods Funct. Theory 20(1), 111-124 (2020)

5. Hu, X.-M., Tian, J.-F., Chu, Y.-M., Xu, Y.-X.: On Cauchy-Schwarz inequality for N-tuple diamond-alpha integral. J. Inequal. Appl. 2020, Article ID 8 (2020) 
6. Yang, Z.-H., Qian, W.-M., Zhang, W., Chu, Y.-M.: Notes on the complete elliptic integral of the first kind. Math. Inequal. Appl. 23(1), 77-93 (2020)

7. Rafeeq, S., Kalsoom, H., Hussain, S., Rashid, S., Chu, Y.-M.: Delay dynamic double integral inequalities on time scales with applications. Adv. Differ. Equ. 2020, Article ID 40 (2020)

8. Abbas Baloch, I., Chu, Y.-M.: Petrović-type inequalities for harmonic $h$-convex functions. J. Funct. Spaces 2020, Article ID 3075390 (2020)

9. Qian, W.-M., He, Z.-Y., Chu, Y.-M.: Approximation for the complete elliptic integral of the first kind. Rev. R. Acad. Cienc. Exactas Fís. Nat., Ser. A Mat. 114(2), Article ID 57 (2020). https://doi.org/10.1007/s13398-020-00784-9

10. Wang, B., Luo, C.-L., Li, S.-H., Chu, Y.-M.: Sharp one-parameter geometric and quadratic means bounds for the Sándor-Yang means. Rev. R. Acad. Cienc. Exactas Fís. Nat., Ser. A Mat. 114(1), Article ID 7 (2020). https://doi.org/10.1007/s13398-019-00734-0

11. Latif, M.A., Rashid, S., Dragomir, S.S., Chu, Y.-M.: Hermite-Hadamard type inequalities for co-ordinated convex and quasi-convex functions and their applications. J. Inequal. Appl. 2019, Article ID 317 (2019)

12. Zaheer Ullah, S., Adil Khan, M., Chu, Y.-M.: A note on generalized convex functions. J. Inequal. Appl. 2019, Article ID $291(2019)$

13. Qian, W.-M., Yang, Y.-Y., Zhang, H.-W., Chu, Y.-M.: Optimal two-parameter geometric and arithmetic mean bounds for the Sándor-Yang mean. J. Inequal. Appl. 2019, Article ID 287 (2019)

14. Wang, M.-K., Chu, H.-H., Chu, Y.-M.: Precise bounds for the weighted Hölder mean of the complete $p$-elliptic integrals. J. Math. Anal. Appl. 480(2), Article ID 123388 (2019). https://doi.org/10.1016/j.jmaa.2019.123388

15. Adil Khan, M., Hanif, M., Khan, Z.A., Ahmad, K., Chu, Y.-M.: Association of Jensen's inequality for s-convex function with Csiszár divergence. J. Inequal. Appl. 2019, Article ID 162 (2019)

16. Zaheer Ullah, S., Adil Khan, M., Khan, Z.A., Chu, Y.-M.: Integral majorization type inequalities for the functions in the sense of strong convexity. J. Funct. Spaces 2019, Article ID 9487823 (2019)

17. Wang, M.-K., Chu, Y.-M., Zhang, W.: Monotonicity and inequalities involving zero-balanced hypergeometric function. Math. Inequal. Appl. 22(2), 601-617 (2019)

18. Yang, Z.-H., Chu, Y.-M., Zhang, W.: High accuracy asymptotic bounds for the complete elliptic integral of the second kind. Appl. Math. Comput. 348, 552-564 (2019)

19. Yang, Z.-H., Qian, W.-M., Chu, Y.-M.: Monotonicity properties and bounds involving the complete elliptic integrals of the first kind. Math. Inequal. Appl. 21(4), 1185-1199 (2018)

20. Song, Y.-Q., Adil Khan, M., Zaheer Ullah, S., Chu, Y.-M.: Integral inequalities involving strongly convex functions. J. Funct. Spaces 2018, Article ID 6595921 (2018)

21. Li, X.-F., Tang, G.-J., Tang, B.-Q.: Stress field around a strike-slip fault in orthotropic elastic layers via a hypersingular integral equation. Comput. Math. Appl. 66(11), 2317-2326 (2013)

22. Huang, C.-X., Guo, S., Liu, L.-Z.: Boundedness on Morrey space for Toeplitz type operator associated to singular integral operator with variable Calderón-Zygmund kernel. J. Math. Inequal. 8(3), 453-464 (2014)

23. Huang, C.-X., Yang, Z.-C., Yi, T.-S., Zou, X.-F.: On the basins of attraction for a class of delay differential equations with non-monotone bistable nonlinearities. J. Differ. Equ. 256(7), 2101-2114 (2014)

24. Xie, D.-X., Li, J.: A new analysis of electrostatic free energy minimization and Poisson-Boltzmann equation for protein in ionic solvent. Nonlinear Anal., Real World Appl. 21, 185-196 (2015)

25. Liu, Y.-C., Wu, J.: Multiple solutions of ordinary differential systems with min-max terms and applications to the fuzzy differential equations. Adv. Differ. Equ. 2015, Article ID 379 (2015)

26. Zhou, W.-J., Wang, F.: A PRP-based residual method for large-scale monotone nonlinear equations. Appl. Math. Comput. 261, 1-7 (2015)

27. Dai, Z.-F:. Comments on a new class of nonlinear conjugate gradient coefficients with global convergence properties. Appl. Math. Comput. 276, 297-300 (2016)

28. Li, J.-L., Sun, G.-Y., Zhang, R.-M.: The numerical solution of scattering by infinite rough interfaces based on the integral equation method. Comput. Math. Appl. 71(7), 1491-1502 (2016)

29. $\mathrm{Hu}, \mathrm{H} .-\mathrm{J}$, Liu, L.-Z.: Weighted inequalities for a general commutator associated to a singular integral operator satisfying a variant of Hörmander's condition. Math. Notes 101(5-6), 830-840 (2017)

30. Cai, Z.-W., Huang, J.-H., Huang, L.-H.: Generalized Lyapunov-Razumikhin method for retarded differential inclusions: applications to discontinuous neural networks. Discrete Contin. Dyn. Syst. 22B(9), 3591-3614 (2017)

31. Wang, W.-S.: On A-stable one-leg methods for solving nonlinear Volterra functional differential equations. Appl. Math. Comput. 314, 380-390 (2017)

32. Peng, J., Zhang, Y.: Heron triangles with figurate number sides. Acta Math. Hung. 157(2), 478-488 (2019)

33. Tian, Z.-L., Liu, Y., Zhang, Y., Liu, Z.-Y., Tian, M.-Y.: The general inner-outer iteration method based on regular splittings for the PageRank problem. Appl. Math. Comput. 356, 479-501 (2019)

34. Wang, W.-S., Chen, Y.-Z., Fang, H.: On the variable two-step IMEX BDF method for parabolic integro-differential equations with nonsmooth initial data arising in finance. SIAM J. Numer. Anal. 57(3), 1289-1317 (2019)

35. Rashid, S., Jarad, F., Chu, Y.-M.: A note on reverse Minkowski inequality via generalized proportional fractional integral operator with respect to another function. Math. Probl. Eng. 2020, Article ID 7630260 (2020)

36. Rashid, S., Jarad, F., Kalsoom, H., Chu, Y.-M.: On Pólya-Szegö and Ćebyšev type inequalities via generalized $k$-fractional integrals. Adv. Differ. Equ. 2020, Article ID 125 (2020)

37. Qian, W.-M., Zhang, W., Chu, Y.-M.: Bounding the convex combination of arithmetic and integral means in terms of one-parameter harmonic and geometric means. Miskolc Math. Notes 20(2), 1157-1166 (2019)

38. Khan, S., Adil Khan, M., Chu, Y.-M.: Converses of the Jensen inequality derived from the Green functions with applications in information theory. Math. Methods Appl. Sci. 43(5), 2577-2587 (2020)

39. Qian, W.-M., He, Z.-Y., Zhang, H.-W., Chu, Y.-M.: Sharp bounds for Neumann means in terms of two-parameter contraharmonic and arithmetic mean. J. Inequal. Appl. 2019, Articel ID 168 (2019)

40. He, X.-H., Qian, W.-M., Xu, H.-Z., Chu, Y.-M.: Sharp power mean bounds for two Sándor-Yang means. Rev. R. Acad. Cienc. Exactas Fís. Nat., Ser. A Mat. 113(3), 2627-2638 (2019)

41. Wang, M.-K., Chu, Y.-M., Zhang, W.: Precise estimates for the solution of Ramanujan's generalized modular equation Ramanujan J. 49(3), 653-668 (2019) 
42. Xia, W.-F., Li, Y.-M., Chu, Y.-M., Xu, S.-Y., Chen, W.-M., Zhang, Z.-Q.: Observer-based mixed passive and $H_{\infty}$ control for uncertain Markovian jump systems with time delays using quantized measurements. Nonlinear Anal. Hybrid Syst. 31, 233-246 (2019)

43. Xia, W.-F., Xu, S.-Y., Luo, J.-W., Zhang, Z.-Q., Chu, Y.-M.: Reliable filter design for discrete-time neural networks with Markovian jumping parameters and time-varying delay. J. Franklin Inst. 357(5), 2892-2915 (2020)

44. Hai, G.-J., Zhao, T.-H.: Monotonicity properties and bounds involving the two-parameter generalized Grötzsch ring function. J. Inequal. Appl. 2020, Article ID 66 (2020)

45. Chu, Y.-M., Wang, M.-K., Qiu, S.-L.: Optimal combinations bounds of root-square and arithmetic means for Toader mean. Proc. Indian Acad. Sci. Math. Sci. 122(1), 41-51 (2012)

46. Wang, M.-K., Zhang, W., Chu, Y.-M.: Monotonicity, convexity and inequalities involving the generalized elliptic integrals. Acta Math. Sci. 39B(5), 1440-1450 (2019)

47. Fang, X.-P., Deng, Y.-J., Li, J.: Plasmon resonance and heat generation in nanostructures. Math. Methods Appl. Sci. 38(18), 4663-4672 (2015)

48. Duan, L., Huang, L.-H., Guo, Z.-Y., Fang, X.-W.: Periodic attractor for reaction-diffusion high-order Hopfield neural networks with time-varying delays. Comput. Math. Appl. 73(2), 233-245 (2017)

49. Wang, W.-S., Chen, Y.-Z.: Fast numerical valuation of options with jump under Merton's model. J. Comput. Appl. Math. 318, 79-92 (2017)

50. Huang, C.-X., Liu, L.-Z.: Boundedness of multilinear singular integral operator with a non-smooth kernel and mean oscillation. Quaest. Math. 40(3), 295-312 (2017)

51. Tan, Y.-X., Huang, C.-X., Sun, B., Wang, T.: Dynamics of a class of delayed reaction-diffusion systems with Neumann boundary condition. J. Math. Anal. Appl. 458(2), 1115-1130 (2018)

52. Tang, W.-S., Zhang, J.-J.: Symplecticity-preserving continuous-stage Runge-Kutta-Nyström methods. Appl. Math. Comput. 323, 204-219 (2018)

53. Duan, L., Fang, X.-W., Huang, C.-X.: Global exponential convergence in a delayed almost periodic Nicholson's blowflies model with discontinuous harvesting. Math. Methods Appl. Sci. 41(5), 1954-1965 (2018)

54. Liu, Z.-Y., Wu, N.-C., Qin, X.-R., Zhang, Y.-L.: Trigonometric transform splitting methods for real symmetric Toeplitz systems. Comput. Math. Appl. 75(8), 2782-2794 (2018)

55. Wang, J.-F., Chen, X.-Y., Huang, L.-H.: The number and stability of limit cycles for planar piecewise linear systems of node-saddle type. J. Math. Anal. Appl. 469(1), 405-427 (2019)

56. Wang, J.-F., Huang, C.-X., Huang, L.-H.: Discontinuity-induced limit cycles in a general planar piecewise linear system of saddle-focus type. Nonlinear Anal. Hybrid Syst. 33, 162-178 (2019)

57. Rashid, S., Ashraf, R., Noor, M.A., Noor, K.I., Chu, Y.-M.: New weighted generalizations for differentiable exponentially convex mapping with application. AIMS Math. 5(4), 3525-3546 (2020)

58. Rashid, S., Noor, M.A., Noor, K.I., Chu, Y.-M.: Ostrowski type inequalities in the sense of generalized $\mathcal{K}$-fractional integral operator for exponentially convex functions. AIMS Math. 5(3), 2629-2645 (2020)

59. Adil Khan, M., Zaheer Ullah, S., Chu, Y.-M.: The concept of coordinate strongly convex functions and related inequalities. Rev. R. Acad. Cienc. Exactas Fís. Nat., Ser. A Mat. 113(3), 2235-2251 (2019)

60. Zaheer Ullah, S., Adil Khan, M., Chu, Y.-M.: Majorization theorems for strongly convex functions. J. Inequal. Appl. 2019, Article ID 58 (2019)

61. Wu, S.-H., Chu, Y.-M.: Schur m-power convexity of generalized geometric Bonferroni mean involving three parameters. J. Inequal. Appl. 2019, Article ID 57 (2019)

62. Adil Khan, M., Wu, S.-H., Ullah, H., Chu, Y.-M.: Discrete majorization type inequalities for convex functions on rectangles. J. Inequal. Appl. 2019, Article ID 16 (2019)

63. Khurshid, Y., Adil Khan, M., Chu, Y.-M.: Conformable integral inequalities of the Hermite-Hadamard type in terms of GG- and GA-convexities. J. Funct. Spaces 2019, Article ID 6926107 (2019)

64. Khurshid, Y., Adil Khan, M., Chu, Y.-M., Khan, Z.A.: Hermite-Hadamard-Fejér inequalities for conformable fractional integrals via preinvex functions. J. Funct. Spaces 2019, Article ID 3146210 (2019)

65. Chu, Y.-M., Adil Khan, M., Ali, T., Dragomir, S.S.: Inequalities for $\alpha$-fractional differentiable functions. J. Inequal. Appl. 2017, Article ID 93 (2017)

66. Hadamard, J.: Étude sur les propriétés des fonctions entières et en particulier d'une fonction considérée par Riemann. J. Math. Pures Appl. 58, 171-215 (1893)

67. Adil Khan, M., Mohammad, N., Nwaeze, E.R., Chu, Y.-M.: Quantum Hermite-Hadamard inequality by means of a Green function. Adv. Differ. Equ. 2020, Article ID 99 (2020)

68. Iqbal, A., Adil Khan, M., Ullah, S., Chu, Y.-M.: Some new Hermite-Hadamard-type inequalities associated with conformable fractional integrals and their applications. J. Funct. Spaces 2020, Article ID 9845407 (2020)

69. Adil Khan, M., Khurshid, Y., Du, T.-S., Chu, Y.-M.: Generalization of Hermite-Hadamard type inequalities via conformable fractional integrals. J. Funct. Spaces 2018, Article ID 5357463 (2018)

70. Adil Khan, M., Iqbal, A., Suleman, M., Chu, Y.-M.: Hermite-Hadamard type inequalities for fractional integrals via Green's function. J. Inequal. Appl. 2018, Article ID 161 (2018)

71. Adil Khan, M., Chu, Y.-M., Kashuri, A., Liko, R., Ali, G.: Conformable fractional integrals versions of Hermite-Hadamard inequalities and their generalizations. J. Funct. Spaces 2018, Article ID 6928130 (2018)

72. Guessab, A., Schmeisser, G.: Sharp integral inequalities of the Hermite-Hadamard type. J. Approx. Theory 115(2), 260-288 (2002)

73. İşcan, I.: Hermite-Hadamard type inequalities for harmonically convex functions. Hacet. J. Math. Stat. 43(6), 935-942 (2014)

74. Toplu, T., Kadakal, M., İscan, İ.: On n-polynomial convexity and some related inequalities. AIMS Math. 5(2), 1304-1318 (2020) 